\title{
Salivary Cortisol does not Correlate with Metabolic Syndrome Markers or Subjective Stress in Overweight Children
}

\section{Strait RB ${ }^{1}$, Slattery $\mathrm{MJ}^{2}{ }^{2}$, Carrel $\mathrm{AL}^{1}$, Eickhoff $\mathrm{J}^{1}$ and Allen $\mathrm{DB}^{1 *}$}

${ }^{1}$ Department of Pediatrics, Division of Endocrinology and Diabetes, University of Wisconsin, School of Medicine and Public Health, 600 Highland Ave, H4-448 CSC, Madison, WI, USA

${ }^{2}$ Department of Child and Adolescent Psychiatry, University of Wisconsin, School of Medicine and Public Health, 6001 Research Park Blvd, Madison, WI, USA

${ }^{*}$ Corresponding author: Allen DB, Department of Pediatrics, Division of Endocrinology and Diabetes, University of Wisconsin, School of Medicine and Public Health, 600 Highland Ave, H4-448 CSC, Madison, WI, USA, Tel: 608-263-9059; Fax: 608-265-7957; E-mail: dballen@wisc.edu

Received date: March 30, 2018; Accepted date: April 12, 2018; Published date: April 18, 2018

Citation: Strait RB, Slattery MJ, Carrel AL, Eickhoff J, Allen DB (2018) Salivary cortisol does not correlate with metabolic syndrome markers or subjective stress in overweight children. J Child Obes Vol No 3 Iss No 2: 8.

Copyright: (c) 2018 Allen DB, et al. This is an open-access article distributed under the terms of the Creative Commons Attribution License, which permits unrestricted use, distribution and reproduction in any medium, provided the original author and source are credited.

\section{Abstract}

Objective: Being overweight is a risk factor for metabolic syndrome in children, but not all overweight children develop metabolic syndrome. Cortisol excess from chronic psychological stress has been proposed as an independent risk factor for metabolic syndrome in this already at-risk population.

The present study assesses the relationship of biochemical and body composition radiographic markers of metabolic syndrome to salivary cortisol and self-reports of chronic psychological stress in a cohort of overweight children.

Methods: This prospective, cross-sectional study took place in a multi-disciplinary pediatric obesity clinic at a tertiary care hospital, and involved fifteen children with BMI at or above the $85^{\text {th }}$ percentile for age and sex, 10 of whom provided salivary cortisol samples. The main outcomes measured were salivary bedtime cortisol, first-waking cortisol, and cortisol awakening response (CAR-the rise in cortisol in the first half hour after waking); fasting serum triglycerides, HDL cholesterol, glucose and insulin for HOMA-IR; the ratio of abdominal fat to total body fat by DXA scan; and scores of validated stress and bullying questionnaires (PANAS-C, PSS, and SEC-Q).

Results: In this pilot study, no correlation was found between salivary cortisol measures and questionnaire scores of subjective stress or bullying, and no correlation was found between any of these measures and markers of metabolic syndrome (dyslipidemia, insulin resistance, increased abdominal fat).

Conclusions: These results suggest that measures of psychological stress, whether biochemical or subjective, do not appear to predict risk of metabolic syndrome in overweight children. While ease of collection and demonstrated utility both in detection of pediatric Cushing disease and in adult psychological research make salivary cortisol assessment an attractive clinical tool, further investigation into the value of salivary measures in pediatric stress research is needed.

Keywords: Salivary cortisol; Psychological stress; Chronic hypercortisolemia; Metabolic syndrome; CAR (Cortisol awakening response)

\section{Introduction}

Pediatric obesity is leading to a significant increase in earlyonset type 2 diabetes, non-alcoholic fatty liver disease, and other metabolic derangements, and these in turn strongly correlate with risk of cardiovascular disease in adulthood [1]. It is poorly understood, however, why some children experience greater morbidity than others from similar degrees of adiposity.

Obesity as defined by body mass index (BMI) does not distinguish between differences in body composition and fat distribution, and thus is an inaccurate predictor of insulin resistance, fatty liver disease, and other components of the metabolic syndrome (MetS). In both obese and non-obese children [2,3] adipose tissue located in ectopic sites (e.g. liver, viscera, muscle) predicts insulin resistance and risk for type 2 diabetes better than BMI. Differential ectopic vs. subcutaneous deposition of fat is influenced by ethnicity [4] genetic polymorphisms such as PNPLA [3,5] and psychological states such as depression in both adolescents and adults [6-8].

Psychological stress leading to chronic amplification of the hypothalamic-pituitary-adrenal (HPA) axis has been proposed as another potential factor promoting deposition of fat into viscera and other ectopic sites, thereby increasing the risk for insulin resistance, hepatic steatosis and dyslipidemia $[9,10]$. While precise mechanisms remain poorly understood, chronic cortisol elevation increases central (especially visceral) adipose tissue deposition while peripheral subcutaneous depots recede [11]. Visceral fat has more inflammatory cells (macrophages) than 
subcutaneous fat [12] and more actively produces adipokines [13] that contribute to a chronic inflammatory state and insulin resistance.

An association between psychological stress, chronic amplification of "stress physiology" (i.e. HPA axis activity, sympatho-adrenal medullary activity, and cardiac autonomic activity), and metabolic syndrome is supported primarily by animal and adult human studies, some of which also demonstrate reversibility [14-17]. A small number of pediatric studies show variable relationships between chronic psychological stress and obesity [18-24]. To our knowledge, no study has explored in a single group the relationship between self-perceived stress, objective bullying, chronic activation of the HPA axis, adipose tissue distribution, and biochemical components of MetS. This study examines the degree to which insulin resistance, dyslipidemia and ectopic fat deposition correlate with chronic psychological stress and salivary cortisol measures of HPA axis activation in overweight children and adolescents.

\section{Methods}

\section{Subjects}

This is a prospective, cross-sectional study of fifteen male and female patients' aged $10-18$ years (average 13.7) with $\mathrm{BMI} \geq 85^{\text {th }}$ percentile, recruited from the University of Wisconsin Pediatric Fitness Clinic. Boys (8) and girls (7) were nearly equally represented, and the racial make-up included African Americans ( 1 male, 2 females), Latino Americans ( 2 males, 1 female), and European Americans (5 boys, 4 girls) in proportion to their relative presence in the local community.

All subjects showed pubertal levels of LH. Exclusion criteria included recent oral or inhaled glucocorticoid therapy, medications for dyslipidemia or blood sugar management, and a diagnosis of diabetes. Ten subjects completed the salivary cortisol portion of the study. The study was reviewed and approved by the Human Subjects Committee Institutional Review Board (IRB) of the University of Wisconsin-Madison School of Medicine and Public Health.

\section{Measurements}

Height was measured with a wall-mounted stadiometer to the nearest $0.1 \mathrm{~cm}$. Weight was measured on a calibrated beam balance platform scale to the nearest $0.1 \mathrm{~kg}$. BMI z-score (BMI z) and BMI-for-age percentiles were computed using the CDC reference values.

\section{Laboratory}

A fasting morning blood sample was drawn for glucose, insulin, HDL, triglycerides, and LH. All serum labs were performed in the UW Health Sciences Core Laboratory. Insulin was measured by chemiluminescent immunoassay.

Salivary cortisol samples were collected by subjects at home using SARSTEDT Salivette-Blue Cap tubes upon first waking (fasting), 30 minutes after waking (fasting), and again between 9 and 11 PM (at least 3 hours since last food ingestion). Salivary cortisol analysis was performed at Mayo Medical Laboratories.

\section{Imaging}

The ratio of abdominal fat to total body fat was determined via DXA using the Norland XR-800 whole body bone densitometer (Norland Corporation, Ft. Atkinson, Wisconsin USA) and tissue masses were analyzed using software Illuminatus version 4.5.0. Scans were performed by the same investigator, and each scan session was preceded by a calibration routine using multiple quality control phantoms that simulate soft tissue and bone.

Body composition values of total bone, muscle and fat mass, as well as \%FAT were measured, with abdominal fat being calculated using the midriff region extending from the bottom of the ribcage to the top of the pelvis (iliac crest).

\section{Psychological Stress and Bullying Assessment}

Psychological stress was assessed by three measures: The Perceived Stress Scale (PSS) [25], a 10-item self-report measure of stress perception/appraisal [26]; The Positive and Negative Affect Schedule for Children (PANAS-C) [27], a 30-item selfreport completed by both the child and the parent [28]; and the Social Experience Questionnaire (SEQ), a 15-item self-report assessment of bullying, an objective source of stress [29-31]. Questionnaires were administered by clinic staff within a week of the clinic appointment.

\section{Statistical Analysis}

All study outcomes were summarized in terms of means \pm standard deviations. Nonparametric Spearman's rank correlation analyses were conducted to evaluate the associations between markers. Fisher z-transformation was utilized to construct the $95 \%$ confidence intervals for the correlation coefficients. All reported $\mathrm{P}$-values are two-sided and $\mathrm{P}<0.05$ was used to define statistical significance. Statistical analyses were conducted using SAS software version 9.4 (SAS Institute Inc., Cary NC).

\section{Results}

There were no significant correlations of MetS risk markers with either salivary cortisol measures of HPA axis elevation or self-reported chronic stress and bullying (Table 1 ). In addition, salivary cortisol measures of HPA axis activity did not correlate with self-reported chronic stress or bullying.

Lack of correlations persisted when boys and girls were studied separately (data not shown). Separate analyses were made with each of the separate subsections of the SEQ-S (bullying) questionnaire, and lack of significance persisted (results not shown).

Finally, an additional analyses (data not shown) for correlation between the questionnaires showed strong and significant correlation $(+0.82, \quad p<0.001)$ between the two stress 
questionnaires (PSS and PANAS-S), but no correlation between these and the SEQ-S questionnaire.

Table 1: Correlations between self-reported stress, HPA activity, and markers of metabolic syndrome.

\begin{tabular}{|c|c|c|}
\hline Comparison & $\begin{array}{l}\text { Correlation } \\
\text { Coefficient } \\
(95 \% \mathrm{Cl})\end{array}$ & $\begin{array}{l}\mathrm{P} \text { - } \\
\text { value }\end{array}$ \\
\hline Ratio abdominal/total fat vs. waking cortisol & $-0.23(-0.70-0.41)$ & 0.477 \\
\hline Ratio abdominal/total fat vs. CAR & $+0.20(-0.37-0.66)$ & 0.482 \\
\hline Ratio abdominal/total fat $v s$. PSS & $-0.11(-0.64-0.50)$ & 0.737 \\
\hline Ratio abdominal/total fat vs. PANAS-S & $-0.40(-0.77-0.21)$ & 0.180 \\
\hline Ratio abdominal/total fat vs. SEQ-S & $-0.18(-0.67-0.41)$ & 0.542 \\
\hline HOMA-IR vs. waking cortisol & $-0.54(-0.84-0.08)$ & 0.073 \\
\hline HOMA-IR vs. CAR & $+0.46(-0.11-0.79)$ & 0.094 \\
\hline HOMA-IR vs. PSS & $+0.38(-0.27-0.78)$ & 0.224 \\
\hline HOMA-IR vs. PANAS-S & $+0.02(-0.56-0.59)$ & 0.949 \\
\hline HOMA-IR vs. SEQ-S & $+0.22(-0.40-0.71)$ & 0.478 \\
\hline HDL vs. waking cortisol & $-0.08(-0.62-0.52)$ & 0.803 \\
\hline HDL vs. CAR & $-0.13(-0.62-0.43)$ & 0.646 \\
\hline HDL vs. PSS & $-0.08(-0.62-0.52)$ & 0.810 \\
\hline HDL vs. PANAS-S & $+0.31(-0.33-0.74)$ & 0.328 \\
\hline HDL vs. SEQ-S & $-0.02(-0.58-0.56)$ & 0.962 \\
\hline Triglycerides vs. waking cortisol & $-0.43(-0.80-0.21)$ & 0.166 \\
\hline Triglycerides vs. CAR & $+0.49(-0.08-0.80)$ & 0.078 \\
\hline Triglycerides vs. PSS & $+0.16(-0.46-0.67)$ & 0.616 \\
\hline Triglycerides vs. PANAS-S & $-0.05(-0.61-0.54)$ & 0.871 \\
\hline Triglycerides vs. SEQ-S & $+0.03(-0.55-0.59)$ & 0.924 \\
\hline
\end{tabular}

\section{Discussion}

This study found no correlation between markers of metabolic syndrome and either salivary markers of HPA axis activity or selfreports of chronic stress and bullying. The results suggest that any potential correlation between these parameters in overweight pubertal children is not robust enough to manifest in a small sample size. It is also possible that salivary cortisol levels do not correlate with subjective measures of stress in children as well as in adults, perhaps because the HPA axis in children is more resilient to chronic stress, or because the duration of stress has been insufficient to result in chronic alteration of HPA activity. Furthermore, while salivary cortisol is validated for detection of cortisol excess in conditions such as Cushing syndrome, it has not yet been proven to reliably detect lower levels of hypercortisolemia [32] in children and adolescents.

Despite the negative results of this study, further exploration of these relationships is supported by a trend toward significant correlation between salivary cortisol measures and HOMA-IR in this study, as well as findings of other studies. For example, in rats and primates, stress-induced cortisol hyper-secretion increases central fat deposition $[16,17]$ In adult humans, cortisol secretion and self-report of psychological stress have been positively associated with central adiposity [33] and both cardiovascular and inflammatory stress responses have shown correlation with increasing waist circumference [34].

Studies in children show conflicting results [23] and are limited in number, sample size, and scope of parameters studied (e.g. to a single serum cortisol sample $[18,22]$ or to questionnaire data alone $[19,35])$. For example, school-related stressful events were positively associated with visceral and subcutaneous adipose tissue in peri-pubertal Latino girls with high CAR, and not in girls with low CAR [20] but laboratory assessment for MetS were not performed. In overweight (BMI $>85^{\text {th }}$ percentile) Latino youth, markers of MetS (increasing $\mathrm{BMI}$, body fat mass by DXA, abdominal adipose tissue by MRI, fasting lipids, decreasing insulin sensitivity) were associated with higher morning serum cortisol [18] but psychological stress was not assessed. In obese peri-pubertal children, fasting morning serum cortisol and especially 24-hour urine free cortisol were associated with increasing waist circumference and laboratory markers of MetS [21] but body composition and psychological stress were not assessed.

Associations between activation of the HPA axis and psychological stress in children also show variable results depending on testing methods. For instance, in adolescent girls, increasing BMI and central adiposity by DXA scan correlated positively with overnight urine free cortisol, but correlated negatively with mid-day pre- and post-venipuncture serum cortisol (controlled stress event) [24]. An explanation offered for these divergent findings is that chronic stress-induced elevation of baseline diurnal activity of the HPA axis eventually leads to blunting of acute reactivity [36]. On the other hand, in a longitudinal study of adolescents, increasing BMI was associated with decreases in salivary cortisol at all times of the day [37]. Further, in a large cohort of obese children and adolescents, while a weak correlation was found between fasting morning serum cortisol and laboratory markers of MetS, no correlation was found with waist circumference [22].

The measurement of salivary cortisol is now considered a feasible, reliable, and accurate indicator of serum free cortisol, and late-night salivary sampling has emerged as a valuable screening test for hyperactivity of the HPA axis in suspected Cushing disease and other states of cortisol excess in adults and children [38]. Salivary cortisol measurement for stress research especially in adults has also expanded significantly in recent years [36,39-41] but while its use has also become more prevalent in pediatric research, the interpretation of both the awakening salivary cortisol and CAR in children has not been reliably established [32]. Self-reported stress in adults is associated with both increased salivary cortisol upon first wakening and CAR [36] but the association in adults of waist circumference with both wakening salivary cortisol and CAR has been conflicting, with some finding a positive correlation [42] and some finding no correlation [43].

This study was limited by small sample size; an expanded study would provide more power to discern subtler associations 
between chronic stress, HPA axis activation, and markers of MetS. Furthermore, while the study population included children with ethnic and economic characteristics that have been associated with stress, it is reasonable to speculate that levels of stress in this study population-organized and motivated enough to attend obesity clinic-were insufficient to illuminate associations. Finally, while the questionnaires used have all been validated for this type of research, simultaneous use of three questionnaires with different Likert scales (e.g. from 0-4 vs. from 1-5), time periods assessed (e.g. over the past 1 week vs. 1 month vs. in general), and formats may have been confusing to the subjects. The finding of high correlation between the PSS and PANAS-S, however, suggests that at least these stress questionnaires were in fact accurate gauges of stress in these children.

\section{Conclusion}

In conclusion, while theoretically attractive, precise relationships between psychological stress, activation of the HPA axis, deposition of ectopic fat and risk for metabolic syndrome remain unresolved. Optimal and feasible approaches to measuring perceived stress and HPA axis activation in children also merit further study. If psychological stress in children can be linked to chronic HPA activation and components of MetS including ectopic fat distribution, insulin resistance and dyslipidemia, a potential role for stress-reducing interventions (e.g. mindfulness training) to reduce the morbidity of obesity could emerge.

\section{Funding}

This work was supported by the National Institutes of Health (grant number T32 DK077586); and the Endocrine Fellows Foundation (grant number 133-AAA7577).

\section{Competing and Conflicting Interests}

The authors report no conflicts of interest.

\section{References}

1. Hong YM (2010) Atherosclerotic cardiovascular disease beginning in childhood. Korean Circ J 40: 1-9.

2. Poonawalla $A H$, Sjoberg $B P$, Rehm JL, Hernando $D$, Hines $C D$, et al. (2013) Adipose tissue MRI for quantitative measurement of central obesity. J Magn Reson Imaging 37: 707-716.

3. Rehm JL, Connor EL, Wolfgram PM, Eickhoff JC, Reeder SB, et al. (2014) Predicting hepatic steatosis in a racially and ethnically diverse cohort of adolescent girls. J Pediatr 165: 319-325.

4. Wolfgram PM, Connor EL, Rehm JL, Eickhoff JC, Reeder SB, et al. (2014) Ethnic differences in the effects of hepatic fat deposition on insulin resistance in nonobese middle school girls. Obesity 22: 243-248.

5. Goran MI, Walker R, Le KA, Mahurkar S, Vikman S, et al. (2010) Effects of PNPLA3 on liver fat and metabolic profile in Hispanic children and adolescents. Diabetes 59: 3127-3130.
6. Appelhans BM, Segawa E, Janssen I, Kazlauskaite R, Thurston RC et al. (2014) Employment status, depressive symptoms, and waist circumference change in midlife women: The study of women's health across the nation (SWAN). Ann Epidemiol 24: 187-192.

7. Beydoun MA, Kuczmarski MT, Mason MA, Ling SM, Evans MK, et al. (2009) Role of depressive symptoms in explaining socioeconomic status disparities in dietary quality and central adiposity among US adults: A structural equation modeling approach. Am J Clin Nutr 90: 1084-1095.

8. Dockray S, Susman EJ, Dorn LD (2009) Depression, cortisol reactivity, and obesity in childhood and adolescence. J Adolesc Health 45: 344-350.

9. Chrousos GP (2000) The role of stress and the hypothalamicpituitary-adrenal axis in the pathogenesis of the metabolic syndrome: Neuro-endocrine and target tissue-related causes. Int J Obes 24: S50-S55.

10. Nicolaides NC, Charmandari E, Kino T, Chrousos GP (2017) Stressrelated and circadian secretion and target tissue actions of glucocorticoids: Impact on health. Front Endocrinol 8: 70.

11. Lee MJ, Pramyothin P, Karastergiou K, Fried SK (2014) Deconstructing the roles of glucocorticoids in adipose tissue biology and the development of central obesity. Biochimica et Biophysica Acta (BBA)-Mol Basis Dis 1842: 473-481.

12. Heilbronn LK, Campbell LV (2008) Adipose tissue macrophages, low grade inflammation and insulin resistance in human obesity. Curr Pharm Des 14: 1225-1230.

13. Hamdy O, Porramatikul S, Al-Ozairi E (2006) Metabolic obesity: The paradox between visceral and subcutaneous fat. Curr Diabetes Rev 2: 367-373.

14. Brunner EJ, Hemingway $H$, Walker BR, Page $M$, Clarke $P$, et al. (2002) Adrenocortical, autonomic, and inflammatory causes of the metabolic syndrome: Nested case-control study. Circulation 106: 2659-2665.

15. Wüst S, Federenko I, Hellhammer DH, Kirschbaum C (2000) Genetic factors, perceived chronic stress, and the free cortisol response to awakening. Psychoneuroendocrinology 25: 707-720.

16. Jayo JM, Shively CA, Kaplan JR, Manuck SB (1993) Effects of exercise and stress on body fat distribution in male cynomolgus monkeys. Int J Obes Relat Metab Disord 17: 597-604.

17. Rebuffe-Scrive M, Walsh UA, McEwen B, Rodin J (1992) Effect of chronic stress and exogenous glucocorticoids on regional fat distribution and metabolism. Physiol Behav 52: 583-590.

18. Weigensberg MJ, Toledo-Corral CM, Goran MI (2008) Association between the metabolic syndrome and serum cortisol in overweight Latino youth. J Clin Endocrinol Metab 93: 1372-1378.

19. Holmes ME, Eisenmann JC, Ekkekakis P, Gentile D (2008) Physical activity, stress, and metabolic risk score in 8- to 18 -year-old boys. J Phys Act Health 5: 294-307.

20. Donoho CJ, Weigensberg MJ, Emken BA, Hsu JW, Spruijt-Metz D (2011) Stress and abdominal fat: Preliminary evidence of moderation by the cortisol awakening response in Hispanic peripubertal girls. Obesity 19: 946-952.

21. Reinehr T, Kulle A, Wolters B, Knop C, Lass N, et al. (2014) Relationships between 24-hour urinary free cortisol concentrations and metabolic syndrome in obese children. J Clin Endocrinol Metab 99: 2391-2399. 
22. Guzzetti C, Pilia S, Ibba A, Loche S (2014) Correlation between cortisol and components of the metabolic syndrome in obese children and adolescents. J Endocrinol Invest 37: 51-56.

23. Bai S, Robles TF, Reynolds BM, Repetti RL (2017) Children's diurnal cortisol responses to negative events at school and home. Psychoneuroendocrinology 83: 150-158.

24. Hillman JB, Dorn LD, Loucks TL, Berga SL (2012) Obesity and the hypothalamic-pituitary-adrenal axis in adolescent girls. Metabolism 61: 341-348.

25. Cohen S, Kamarck T, Mermelstein R (1983) A global measure of perceived stress. J Health Soc Behav 24: 385-396.

26. Slattery MJ, Grieve AJ, Ames ME, Armstrong JM, Essex MJ (2013) Neurocognitive function and state cognitive stress appraisal predict cortisol reactivity to an acute psychosocial stressor in adolescents. Psychoneuroendocrinology 38: 1318-1327.

27. Ebesutani C, Okamura K, Higa-McMillan C, Chorpita BF (2011) A psychometric analysis of the positive and negative affect schedule for children-parent version in a school sample. Psychol Assess 23: 406-416.

28. Buchanan TW, al'Absi M, Lovallo WR (1999) Cortisol fluctuates with increases and decreases in negative affect. Psychoneuroendocrinology 24: 227-241.

29. Desjardins T, Yeung Thompson RS, Sukhawathanakul P, Leadbeater BJ, Macdonald SW (2013) Factor structure of the Social Experience Questionnaire across time, sex, and grade among early elementary school children. Psychol Assess 25: 1058-1068.

30. Storch EA, Crisp H, Roberti JW, Bagner DM, Masia-Warner C (2005) Psychometric evaluation of the social experience questionnaire in adolescents: Descriptive data, reliability, and factorial validity. Child Psychiatry Hum Dev 36: 167-176.

31. Crick NR, Grotpeter JK (1996) Children's treatment by peers: Victims of relational and overt aggression. Dev Psychopathol 8: 367-380.

32. Michels N, Sioen I, De Vriendt T, Huybrechts I, Vanaelst BA, et al. (2012) Children's morning and evening salivary cortisol: Pattern, instruction compliance and sampling confounders. Horm Res Paediatr 77: 27-35.
33. Epel ES, McEwen B, Seeman T, Matthews K, Castellazzo G, et al. (2000) Stress and body shape: Stress-induced cortisol secretion is consistently greater among women with central fat. Psychosom Med 62: 623-632.

34. Brydon L (2011) Adiposity, leptin and stress reactivity in humans. Biol Psychol 86: 114-120.

35. Suchday S, Bellehsen M, Friedberg JP, Almeida M, Kaplan E (2014) Clustering of cardiac risk factors associated with the metabolic syndrome and associations with psychosocial distress in a young Asian Indian population. J Behav Med 37: 725-735.

36. Fries E, Dettenborn L, Kirschbaum C (2009) The cortisol awakening response (CAR): Facts and future directions. Int J Psychophysiol 72: 67-73.

37. Ruttle PL, Javaras KN, Klein MH, Armstrong JM, Burk LR, et al. (2013) Concurrent and longitudinal associations between diurnal cortisol and body mass index across adolescence. J Adolesc Health 52: 731-737.

38. Raff H (2013) Update on late-night salivary cortisol for the diagnosis of Cushing's syndrome: Methodological considerations. Endocrine 44: 346-349.

39. Kirschbaum C, Hellhammer DH (1989) Salivary cortisol in psychobiological research: An overview. Neuropsychobiology 22: 150-169.

40. Bosch JA (2014) The use of saliva markers in psychobiology: Mechanisms and methods. Monogr Oral Sci 24: 99-108.

41. Adam EK, Kumari M (2009) Assessing salivary cortisol in largescale, epidemiological research. Psychoneuroendocrinology 34: 1423-1436.

42. Champaneri S, Xu X, Carnethon MR, Bertoni AG, Seeman T, et al. (2013) Diurnal salivary cortisol is associated with body mass index and waist circumference: The multiethnic study of atherosclerosis. Obesity 21: E56-E63.

43. Kumari M, Chandola T, Brunner E, Kivimaki M (2010) A nonlinear relationship of generalized and central obesity with diurnal cortisol secretion in the Whitehall II study. J Clin Endocrinol Metab 95: 4415-4423. 